\title{
THE NUMBER OF DISTINCT EIGENVALUES OF A MATRIX AFTER PERTURBATION*
}

\author{
P. E. FARRELL ${ }^{\dagger}$
}

\begin{abstract}
We prove a new theorem relating the number of distinct eigenvalues of a matrix after perturbation to the prior number of distinct eigenvalues, the rank of the update, and the degree of nondiagonalizability of the matrix. In particular, a rank one update applied to a diagonalizable matrix can at most double the number of distinct eigenvalues. The theorem applies to both symmetric and nonsymmetric matrices and perturbations, of arbitrary magnitudes. An an application, we prove that in exact arithmetic the number of Krylov iterations required to exactly solve a linear system involving a diagonalizable matrix can at most double after a rank one update.
\end{abstract}

Key words. distinct eigenvalues, perturbation, Krylov methods, deflation

AMS subject classifications. 15A18, 65F15, 65F10

DOI. $10.1137 / 15 \mathrm{M} 1037603$

1. Distinct eigenvalues after perturbation. The spectrum of a matrix after perturbation is of interest in a wide variety of applications and has been studied extensively in various particular cases, with most work focusing on the case of symmetric rank one perturbations $[16,5,3,9]$. More general results concern the Jordan form of the matrix after "generic" rank one perturbations, i.e., the set of rank one perturbations for which the analysis does not hold has Lebesgue measure zero $[7,15,11,10]$. In this work we prove a new theorem regarding the number of distinct eigenvalues of arbitrary matrices perturbed by updates of arbitrary rank.

Let $\Lambda(M)$ be the set of distinct eigenvalues of a matrix $M$. Let $m_{a}(M, \lambda)$ and $m_{g}(M, \lambda)$ be the algebraic and geometric multiplicity of $\lambda$ as an eigenvalue of $M$, respectively.

DeFINITION 1.1 (defectivity of an eigenvalue). The defectivity of an eigenvalue $d(M, \lambda) \geq 0$ is the difference between its algebraic and geometric multiplicities,

$$
d(M, \lambda) \equiv m_{a}(M, \lambda)-m_{g}(M, \lambda) .
$$

Definition 1.2 (defectivity of a matrix). The defectivity of a matrix $d(M)$ is the sum of the defectivities of its eigenvalues:

$$
d(M) \equiv \sum_{\lambda \in \Lambda(M)}\left(m_{a}(M, \lambda)-m_{g}(M, \lambda)\right) .
$$

Recall that $m_{a}(M, \lambda) \geq m_{g}(M, \lambda)$ for all $M$ and $\lambda$. Thus, $d(M, \lambda) \geq 0$, and $d(M) \geq 0$. Defectivity is a quantitative measure of nondiagonalizability: a matrix is diagonalizable if and only if it has defectivity zero.

Remark 1. The defectivity of a matrix is clear from its Jordan form: it is the number of off-diagonal ones.

* Received by the editors August 31, 2015; accepted for publication (in revised form) by D. Orban March 3, 2016; published electronically April 26, 2016. The research of the author was funded by EPSRC grants EP/K030930/1, EP/M019721/1, and a Center of Excellence grant from the Research Council of Norway to the Center for Biomedical Computing at Simula Research Laboratory.

http://www.siam.org/journals/simax/37-2/M103760.html

${ }^{\dagger}$ Mathematical Institute, University of Oxford, Oxford, OX2 6GG, UK, and Center for Biomedical Computing, Simula Research Laboratory, Oslo, Norway (patrick.farrell@maths.ox.ac.uk). 
We now give the central theorem of this paper.

Theorem 1.3. Let $A, B \in \mathbb{C}^{n \times n}$. If $C=A+B$, then $|\Lambda(C)| \leq(\operatorname{rank}(B)+$ 1) $|\Lambda(A)|+d(A)$.

Proof. Clearly $|\Lambda(C)|=|\Lambda(C) \cap \Lambda(A)|+|\Lambda(C) \backslash \Lambda(A)|$, and the first term is bounded by $|\Lambda(A)|$. We seek an upper bound for the quantity

$$
\sum_{\substack{\lambda \in \Lambda(C) \\ \lambda \notin \Lambda(A)}} m_{a}(C, \lambda)
$$

as this bounds the number of new eigenvalues that the perturbation can introduce. (Every eigenvalue $\lambda$ of $C$ must have $m_{a}(C, \lambda) \geq 1$.) Since for $M \in \mathbb{R}^{n \times n}$,

$$
\sum_{\lambda \in \Lambda(M)} m_{a}(M, \lambda)=n
$$

it follows that

$$
\sum_{\substack{\lambda \in \Lambda(C) \\ \lambda \notin \Lambda(A)}} m_{a}(C, \lambda)+\sum_{\lambda \in \Lambda(A)} m_{a}(C, \lambda)=n,
$$

with the convention that $m_{a}(C, \lambda)=0 \Longleftrightarrow \lambda \notin \Lambda(C)$. Thus, the upper bound on the number of new eigenvalues introduced is maximized when

$$
\sum_{\lambda \in \Lambda(A)} m_{a}(C, \lambda)
$$

is minimized.

Let $\lambda \in \Lambda(A)$. We first investigate $m_{g}(C, \lambda)$, the geometric multiplicity of $\lambda$ as an eigenvalue of the perturbed matrix $C$. Using the fact that $\operatorname{rank}(X+Y) \leq$ $\operatorname{rank}(X)+\operatorname{rank}(Y)$, we derive a lower bound for $m_{g}(C, \lambda)$ :

$$
\begin{aligned}
\operatorname{rank}(A+B-\lambda I) & \leq \operatorname{rank}(A-\lambda I)+\operatorname{rank}(B) \\
\Longrightarrow n-\operatorname{dim} \operatorname{ker}(A+B-\lambda I) & \leq n-\operatorname{dim} \operatorname{ker}(A-\lambda I)+\operatorname{rank}(B) \\
\Longrightarrow \quad m_{g}(C, \lambda) & \geq m_{g}(A, \lambda)-\operatorname{rank}(B) .
\end{aligned}
$$

Hence, the geometric multiplicity of an eigenvalue can at most decrease by $r$ on perturbation by a rank- $r$ operator.

It therefore follows that

$\sum_{\lambda \in \Lambda(A)} m_{a}(C, \lambda) \geq \sum_{\lambda \in \Lambda(A)} m_{g}(C, \lambda)$

$$
\begin{array}{ll}
\geq \sum_{\lambda \in \Lambda(A)}\left(m_{g}(A, \lambda)-\operatorname{rank}(B)\right) & \text { (by }(1.7 \mathrm{c})) \\
=\sum_{\lambda \in \Lambda(A)}\left(m_{a}(A, \lambda)-\operatorname{rank}(B)-d(A, \lambda)\right) & \text { (by Definition 1.1) } \\
=n-\operatorname{rank}(B)|\Lambda(A)|-d(A) . &
\end{array}
$$

Copyright $@$ by SIAM. Unauthorized reproduction of this article is prohibited. 
The maximal number of new eigenvalues is achieved when (1.8) is an equality and

$$
\sum_{\substack{\lambda \in \Lambda(C) \\ \lambda \notin \Lambda(A)}} m_{a}(C, \lambda)=n-(n-\operatorname{rank}(B)|\Lambda(A)|-d(A))=\operatorname{rank}(B)|\Lambda(A)|+d(A) .
$$

Hence

$$
\begin{aligned}
|\Lambda(C)| & =|\Lambda(C) \cap \Lambda(A)|+|\Lambda(C) \backslash \Lambda(A)| \\
& \leq|\Lambda(A)|+\operatorname{rank}(B)|\Lambda(A)|+d(A)=(\operatorname{rank}(B)+1)|\Lambda(A)|+d(A) .
\end{aligned}
$$

Corollary 1.4. Let $A$ be diagonalizable (i.e., $d(A)=0$ ) and let $B$ have rank one. If $C=A+B$, then $|\Lambda(C)| \leq 2|\Lambda(A)|$.

2. Krylov iterations after a rank one update. Consider the linear systems $A x=b$ and $C y=d$. If $A$ is diagonalizable, then its minimal polynomial degree $\operatorname{mpd}(A)=|\Lambda(A)|$, and an optimal Krylov method (generalized minimum residual method [14], minimal residual method [13], or conjugate gradients [6], if applicable) will compute $x$ exactly in the same number of iterations. (Here, and henceforth, exact arithmetic is assumed.)

Theorem 2.1. Consider the linear systems $A x=b$ and $C y=d$. Let $A$ be diagonalizable, and let $B$ have rank one. If $C=A+B$, then $y$ can be computed exactly with an optimal Krylov method in at most double the number of iterations required for $x$.

Proof. If $C$ is diagonalizable, then $\operatorname{mpd}(C)=|\Lambda(C)| \leq 2|\Lambda(A)|=2 \operatorname{mpd}(A)$, i.e. the number of distinct eigenvalues bounds the number of Krylov iterations required to solve the perturbed matrix.

If $C$ is not diagonalizable, we know from (1.7c) that the number of Jordan blocks associated with an eigenvalue $\lambda \in \Lambda(A)$ can decrease by at most $1=\operatorname{rank}(B)$ in $C$. Since by diagonalizability of $A$ all its Jordan blocks are of size $1 \times 1$, the largest Jordan block of $C$ can be at most of size $|\Lambda(A)| \times|\Lambda(A)|$, which can occur when all eigenvalues of $A$ lose exactly one Jordan block. It is straightforward to calculate that with any arrangement of new Jordan blocks of $C$ with sizes adding to $|\Lambda(A)|$, the number of Krylov iterations required to compute $y$ is bounded by twice that of $x$.

3. Application: Schur complement preconditioners and deflation. Theorem 1.3 is mainly of interest in situations where $|\Lambda(A)|$ is expected to be small. Such a situation arises in the application of preconditioners based on Schur complements.

Let $F: \mathbb{R}^{n} \rightarrow \mathbb{R}^{n}$ be the (discretized) residual of a nonlinear problem

$$
F(u)=0
$$

with block-structured Jacobian

$$
J=\left[\begin{array}{ll}
X & Y \\
Z & 0
\end{array}\right]
$$

with $X$ invertible. This structure arises in many problems, including the Stokes and Navier-Stokes equations, and in equality-constrained optimization [1]. Linear systems involving $J$ are typically solved with Schur complement preconditioners. Define

$$
P=\left[\begin{array}{cc}
X & 0 \\
0 & -S
\end{array}\right],
$$


where the Schur complement $S=-Z X^{-1} Y$. If $P$ is used as a preconditioner, then the preconditioned operator $P^{-1} J$ is diagonalizable and has exactly three distinct eigenvalues (with exact inner solves for the application of $P^{-1}$ ) [12]. Similar results hold for more general block-structured Jacobians and preconditioners based on the Schur complement: the preconditioned operator has a small number of distinct eigenvalues [8].

Suppose (3.1) supports multiple solutions. One approach to compute them is to initialize Newton's method from many different initial guesses, hoping to start in different basins of convergence. A highly effective alternative is to deflate known solutions [4]. Suppose one solution $u_{1}^{*}$ of (3.1) has been computed from an initial guess $u_{0}$ and additional solutions are sought. We construct a modified residual

$$
G(u)=M\left(u ; u_{1}^{*}\right) F(u)
$$

via the application of a deflation operator $M: \mathbb{R}^{n} \times \mathbb{R}^{n} \rightarrow \mathbb{R}$ to the residual $F$. This deflation operator guarantees two properties. The first is the preservation of solutions of $F$, i.e., for $u \neq u_{1}^{*}, G(u)=0 \Longleftrightarrow F(u)=0$. The second is that Newton's method (or other root finding algorithms) applied to $G$ will not discover $u_{1}^{*}$ again, as

$$
\liminf _{u \rightarrow u_{1}^{*}}\|G(u)\|>0,
$$

i.e., along any sequence converging to the known root, its existence is masked by the nonconvergence of the deflated residual to zero. ( $M$ achieves this by introducing a pole of the appropriate strength at the known solution.) Thus, if Newton's method applied to $G$ converges from $u_{0}$, it will converge to another solution $u_{2}^{*} \neq u_{1}^{*}$. A typical deflation operator is

$$
M\left(u ; u_{1}^{*}\right)=\frac{1}{\left\|u-u_{1}^{*}\right\|^{p}}+1
$$

where $p$ controls the strength of the pole introduced.

The process can then be repeated until no more solutions are found from $u_{0}$ in a fixed number of Newton iterations. Several solutions can be deflated with an operator $M: \mathbb{R}^{n} \times \mathbb{R}^{n} \times \cdots \mathbb{R}^{n} \rightarrow \mathbb{R}$ via

$$
M\left(u ; u_{1}^{*}, \ldots, u_{k}^{*}\right)=\frac{1}{\left\|u-u_{1}^{*}\right\|^{p}} \cdots \frac{1}{\left\|u-u_{k}^{*}\right\|^{p}}+1 .
$$

For full details, see Brown and Gearhart [2] and Farrell, Birkisson, and Funke [4].

The Jacobian $\tilde{J}$ of the deflated problem (3.4) is a rank one update of a scaling of the Jacobian of the original problem (3.1), regardless of the number of solutions deflated:

$$
\tilde{J}=M J+F E^{T},
$$

where $E=M^{\prime} \in \mathbb{R}^{n}$. Hence, the preconditioned deflated Jacobian is also a rank one update of the preconditioned original Jacobian,

$$
C=P^{-1} \tilde{J}=M P^{-1} J+\left(P^{-1} F\right) E^{T}=A+B,
$$

and Theorem 2.1 guarantees that the solutions of linear systems involving the deflated Jacobian can be computed exactly in no more than twice the number of Krylov iterations required for the undeflated Jacobian. 
Acknowledgements. The author would like to thank A. J. Wathen, M. A. Saunders, C. Beentjes, and N. I. M. Gould for useful discussions.

\section{REFERENCES}

[1] M. Benzi, G. H. Golub, And J. Liesen, Numerical solution of saddle point problems, Acta Numer., 14 (2005), pp. 1-137.

[2] K. M. Brown AND W. B. GEARhart, Deflation techniques for the calculation of further solutions of a nonlinear system, Numer. Math., 16 (1971), pp. 334-342.

[3] J. R. Bunch, C. P. Nielsen, And D. C. Sorensen, Rank-one modification of the symmetric eigenproblem, Numer. Math., 31 (1978), pp. 31-48.

[4] P. E. Farrell, A. Birkisson, and S. W. Funke, Deflation techniques for finding distinct solutions of nonlinear partial differential equations, SIAM J. Sci. Comput., 37 (2015), pp. A2026-A2045.

[5] G. H. Golub, Some modified matrix eigenvalue problems, SIAM Rev., 15 (1973), pp. 318-334.

[6] M. R. Hestenes And E. Stiefel, Methods of conjugate gradients for solving linear systems, J. Res. Nat. Bur. Standards, 49 (1952), pp. 409-436.

[7] L. Hörmander And A. Melin, A remark on perturbations of compact operators, Math. Scand., 75 (1994), pp. 255-262.

[8] I. C. F. IPSEN, A note on preconditioning nonsymmetric matrices, SIAM J. Sci. Comput., 23 (2001), pp. 1050-1051.

[9] I. C. F. IPSEN AND B. NADLER, Refined perturbation bounds for eigenvalues of Hermitian and non-Hermitian matrices, SIAM J. Matrix Anal. Appl., 31 (2009), pp. 40-53.

[10] C. Mehl, V. Mehrmann, A. C. M. Ran, and L. Rodman, Jordan forms of real and complex matrices under rank one perturbations, Oper. Matrices, 7 (2013), pp. 381-398.

[11] J. Moro And F. M. Dopico, Low rank perturbation of Jordan structure, SIAM J. Matrix Anal. Appl., 25 (2003), pp. 495-506.

[12] M. F. Murphy, G. H. Golub, and A. J. Wathen, A note on preconditioning for indefinite linear systems, SIAM J. Sci. Comput., 21 (2000), pp. 1969-1972.

[13] C. C. Paige And M. A. Saunders, Solution of sparse indefinite systems of linear equations, SIAM J. Numer. Anal., 12 (1975), pp. 617-629.

[14] Y. SAAd AND M. H. Schultz, GMRES: A generalized minimal residual algorithm for solving nonsymmetric linear systems, SIAM J. Sci. Stat. Comput., 7 (1986), pp. 856-869.

[15] S. V. SAvchenko, Typical changes in spectral properties under perturbations by a rank-one operator, Math. Notes, 74 (2003), pp. 557-0568.

[16] J. H. Wilkinson, The Algebraic Eigenvalue Problem, Monogr. Numer. Anal. 87, Oxford University Press, Oxford, 1965.

Copyright $@$ by SIAM. Unauthorized reproduction of this article is prohibited. 